\title{
How electrostatic and non-electrostatic interactions play a role in water wettability of possible nanostructure surfaces
}

\author{
Nima Novin ${ }^{1} \cdot$ Abolghasem Shameli $^{2}$ (D) Ebrahim Balali ${ }^{1} \cdot$ Shahab Zomorodbakhsh $^{3}$
}

Received: 9 August 2019 / Accepted: 27 December 2019 / Published online: 4 January 2020

(c) The Author(s) 2020

\begin{abstract}
The present paper investigated the effects of the electrostatic and non-electrostatic interactions on the water wettability of a surface. Based on this aim, examinations are performed on the molybdenum disulfide $\left(\mathrm{MoS}_{2}\right)$ as possible nanostructure surface. The results obtained through calculating the water contact angle on the $\mathrm{MoS}_{2}$ surface indicate which this surface is a weak hydrophobic substrate. The present simulations illustrate that the electrostatic interactions have little impact on the wettability amount of the $\mathrm{MoS}_{2}$ substrate. However, the molybdenum disulfide is composed of two charged parts with significant values. In addition, it is observed that the relation between density, van der Waals and electrostatic interactions is different from each other. It have been observed that the van der Waals interactions have direct relationship with water density while there is not anything between the electrostatic interactions and water density peak. The Obtained results via simulation demonstrate that the effectiveness of electrostatic interactions on the wettability depends on the sigma in the Lennard-Jones equation. In the other words, the role of electrostatic and van der Waals interactions on the wettability are not indissociable from each other and this method is sufficient in nanostructure systems.
\end{abstract}

\section{Graphic abstract}

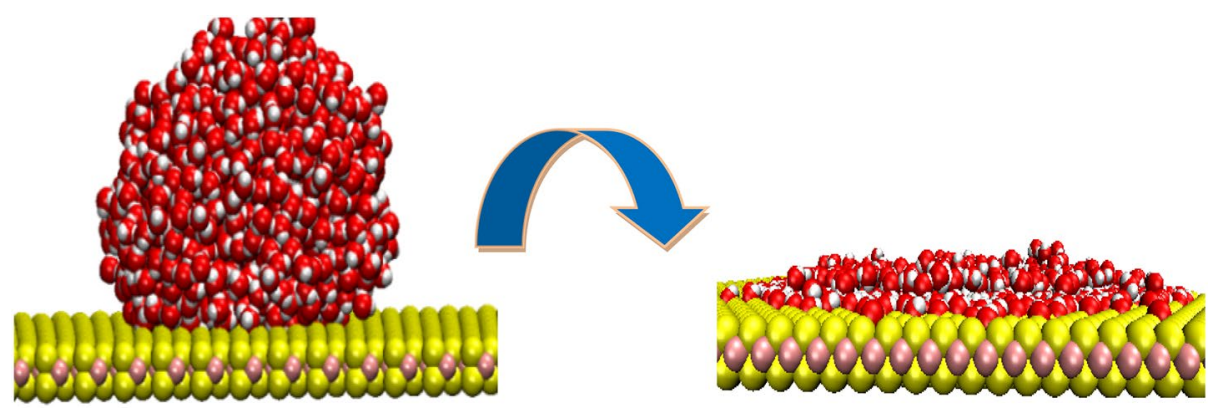

Keywords Molybdenum disulfide $\cdot$ van der Waals interactions $\cdot$ Simulation $\cdot$ Electrostatic $\cdot$ Nano sheets

\section{Introduction}

Abolghasem Shameli

shameli678@gmail.com

1 Department of Chemistry, Faculty of Pharmaceutical Chemistry, Tehran Medical Sciences, Islamic Azad University, Tehran, Iran

2 Department of Chemistry, Faculty of Science, Omidiyeh Branch, Islamic Azad University, Omidiyeh, Iran

3 Department of Chemistry, Faculty of Science, Mahshahr Branch, Islamic Azad University, Mahshahr, Iran
The interface water has a vital role in the biological and microfluidic technologies [1-3]. The interaction between the water molecule and different substrates mainly leads to the changes in the water structure rather than that in bulk structure. According to the substrate tendency to interact with the water molecules, they can be divided into hydrophilic and hydrophobic substrates. The recognition of hydrophilic and hydrophobic surfaces have important 
implications for advancing the industrial goals such as oil separation, lubrication, printing, spray quenching and liquid coating [4-9]. Wettability study is usually associated with the determination of the contact angle indicating how the droplet interacts with the substrate. Small contact angles indicate the hydrophobic surface while larger ones stand for the hydrophilic counterpart.

It is often thought that water wettability increases on the surfaces with components cause of partial charge. This is mainly due to the fact that the water molecules tend to interact with the charged particles. For example, some surfaces such as $\mathrm{TiO}_{2}$ meet strong wettability [10-12] as it is composed of two charged components. Nevertheless, there can be found many surfaces with charged components without strong wettability. The experimental and theoretical results indicate that the $\mathrm{MoS}_{2}$ substrate is a weak hydrophobic surface [13-15]. There are also many substrates having significant hydrophobic properties [16-18].

Recently, a quantum study showed that the only physisorption interactions may arise during the adsorption process of the external molecules such as water on the $\mathrm{MoS}_{2}$ substrate. This has been evidenced by the estimated adsorption energies for the isolated $\mathrm{H}_{2} \mathrm{O}$ physisorption on the monolayer $\mathrm{MoS}_{2}(0.15 \mathrm{eV})$ [19]. Furthermore, the molecular dynamics simulation has illustrated that the electrostatic interaction has little effect on the interaction of water on the $\mathrm{MoS}_{2}$ surface [13]. In addition, the results of this study indicated that the formation of water molecules and their orientation in a water drop causes an electrostatic interaction to become less effective.

Although a large number of researches have been conducted on investigating the interaction impact of various systems on the fluid wettability amount on various substrates, this question still remains unanswered that how much are the contributions of the electrostatic and non-electrostatic interactions to the wettability. The aims of this paper is the studying the interaction of water droplet on the $\mathrm{MoS}_{2}$ surface by use of the molecular dynamics (MD) simulations. The $\mathrm{MoS}_{2}$ surface is used as possible nanostructure substrate with the charged components. At First, the wateriness on the $\mathrm{MoS}_{2}$ surface has been studied. Then, it has been made clear that the influences of the electrostatic and van der Waals interactions on the wettability are quite different from each other.

\section{Simulation details}

The MD simulations were performed by use of LAMMPS [20]. The VMD package visualized the structures [21]. All simulations have been carried out in the NVT ensemble. Nosé-Hoover thermostat [22] was applied to keep at the temperature of $300 \mathrm{~K}$ with damping coefficients as $0.1 \mathrm{ps}^{-1}$. The Verlet algorithm was used for integrating the equations of motion with a time step of $1 \mathrm{fs}$. Non-bonded van der Waals interactions were modeled in terms of 12-6 Lennard-Jones potentials [23]. The particle-particle particle-mesh (PPPM) method to minimize the error in long-range terms in both Coulomb's was applied. Respectively, the Lennard-Jones and Columbic cut-off radiuses were estimated at 10 and $12 \AA$. Each MD simulation was run for $1.0 \mathrm{~ns}$. The SPC/E $[13,24,25]$ water model was implemented here. The simulation box dimensions for a water droplet on molybdenum disulfide surfaces was about $(120 \times 120 \times 100) \AA^{3}$. The water cubic box was initially placed on the top of the molybdenum with the size of $(20 \times 20 \times 16) \AA^{3}$. In both systems, the number of water molecules was 1000 . The substrates were fixed during the simulations. The non-periodic boundary conditions were applied in the $Z$-direction of the simulation box while the periodic ones were considered in the other two directions. In the $Z$ direction (vertical direction), the mirror boundary condition was specified in high surfaces. Non-bonding interaction between components of the system have described via the force field defined in Ref. [13].

\section{Discussion and results}

Intermolecular interactions are divided into two categories including the electrostatic and non-electrostatic ones. The electrostatic and non-electrostatic interactions between the atoms that they involved in the present system are given in Table 1.

Table 1 indicates that the electrostatic and van der Waals interactions have distributed differently among the system components while studying the interaction of water and molybdenum disulfide. As can be seen from Table 1, the electrostatic interactions between the water and substrate ingredients are greater than the van der Waals counterparts.
Table 1 Comparison of the van der Waals and electrostatic interactions between the atoms involved in the studied system (Kcal/mol)

\begin{tabular}{|c|c|c|c|c|c|c|c|c|}
\hline \multicolumn{4}{|c|}{ Electrostatic interaction } & \multicolumn{4}{|c|}{ Van der Waals interaction } & \\
\hline$S$ & Mo & $\mathrm{H}$ & $\mathrm{O}$ & $\mathrm{S}$ & Mo & $\mathrm{H}$ & $\mathrm{O}$ & \\
\hline 7.39805 & -14.7961 & -10.5669 & 21.13384 & 0.39856 & 1.59081 & 0 & 0.32756 & $\mathrm{O}$ \\
\hline-3.6990 & 7.39805 & 5.28346 & -10.5669 & 0 & 0 & 0 & 0 & $\mathrm{H}$ \\
\hline-5.17948 & 10.35897 & 7.39805 & -14.7961 & 27.10470 & 2.91226 & 0 & 1.59081 & Mo \\
\hline 2.58974 & -5.17948 & -3.6990 & 7.39805 & 10.48329 & 27.10470 & 0 & 0.39856 & S \\
\hline
\end{tabular}


However, the fact that how these types of interactions play a role in wettability and how much is the contribution of each interaction is a topic which has not been studied in details. Figure 1 shows the wettability of water droplet on the $\mathrm{MoS}_{2}$ substrate.

The estimated contact angle for the water on $\mathrm{MoS}_{2}$ substrate in this work is $95^{\circ}$ which is inconsistent with the experimental and reported simulated data [13]. Since the contact angle is a benchmark for assessing the interactions between the substrate and fluids, one can investigate the influences of the electrostatic and van der Waal interactions on the wettability through evaluating this angle. For this purpose, in addition to the simulation of the water droplet on $\mathrm{MoS}_{2}$ substrate, several other simulations have been performed here in which the atom charge and sigma related to Lennard-Jones equation have been changed. Although this operations is not empirically justified, but such investigations are commonly carried out in the MD simulations. The aim of this work is to observe how the electrostatic and van der Waals parameters affect the interaction of water with the substrate molybdenum disulfide. Therefore, three other simulations were presented with the following features: (a) the charge of molybdenum and sulfur atoms were considered as zero, (b) sigma related to Lennard-Jones equation between oxygen and sulfur atoms, was considered as $2.147 \AA$, (c) both charges of molybdenum and sulfur atoms and sigma related to Lennard-Jones equation

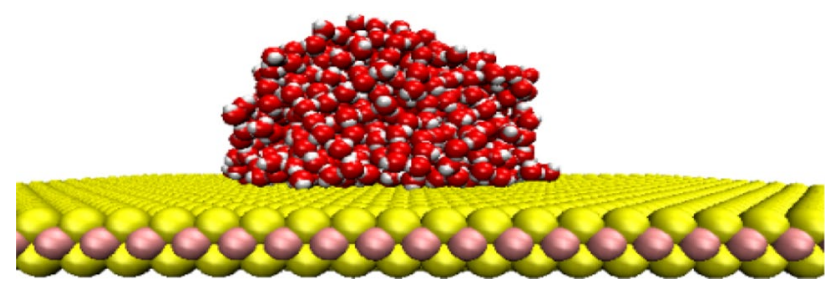

Fig. 1 Water contact angle on $\mathrm{MoS}_{2}$ substrate. The red, white, yellow and pink colors refer to the oxygen, hydrogen, sulfur and molybdenum, respectively between oxygen and sulfur atoms were considered to be 0 and $2.147 \AA$, respectively. Figure 2 shows the water contact angle for the above mentioned systems.

Figure 2 shows that when the charges of Mo and $\mathrm{S}$ atoms are considered as zero (Fig. 2a), the contact angle will be $92^{\circ}$. In fact, this observation indicates that the electrostatic interactions have negligible impact on the wettability amount of the $\mathrm{MoS}_{2}$ substrate. Besides, according to Table 1, the electrostatic interactions between the water molecules and molybdenum disulfide is much more than the van der Waals interactions between them. The reason for this fact will be illustrated in the next sections.

As depicted in Fig. 2b, when the sigma of Lennard-Jones equation between water and sulfur is reduced to $2.147 \AA$, the contact angle will be decreased in $18^{\circ}$, which points out to the strong interaction between water and substrate. Figure $2 \mathrm{c}$ indicates the significant increasing of the water contact angle $\left(114^{\circ}\right)$ when the charges of molybdenum and sulfur atoms and sigma between oxygen and sulfur atoms are considered as 0 and $2.147 \AA$, respectively. This significant trend difference between Fig. $2 b$ and c, stands for the intense effect of the electrostatic interactions between the water and substrate while the slight difference in the water wettability behavior in Fig. 2a and original simulation system (Fig. 1) indicates their small effect. To justify this event, further calculations are required which are described as below.

From the basic calculations which can provide useful information about the behavior of water molecules in the droplets, is the study of the water density variations at different distances from the substrate. Figure 3 depicts the density of water droplet on the $\mathrm{MoS}_{2}$ substrate and a, b and $\mathrm{c}$ systems in the $Z$ direction (perpendicular to the substrate).

Figure 3 shows that the water density on the $\mathrm{MoS}_{2}$ surface has two peaks below $7 \AA$. Comparing to the original system (Fig. 1) and system a, it can be concluded that the partial charge has no notable effect on the density peaks. It should be mentioned that the only difference in the present main system and system a is the partial charges of Mo and $\mathrm{s}$ atoms which they were considered to be zero in the later one. In

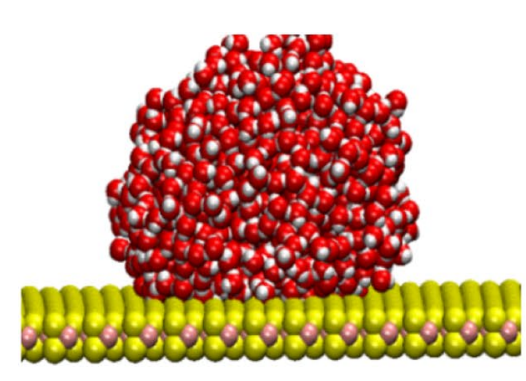

a

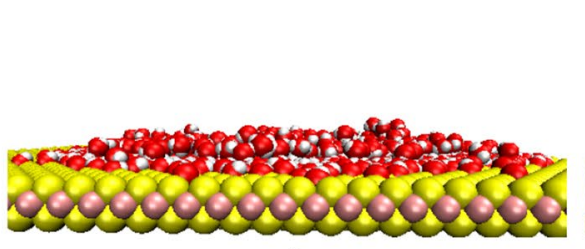

b

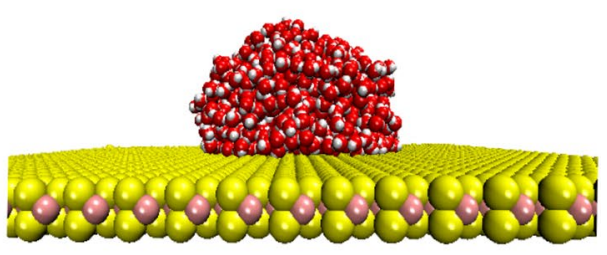

C

Fig. 2 The water contact angle on the three systems defined as systems a, b and c. The red, white, yellow and pink colors refer to the oxygen, hydrogen, sulfur and molybdenum, respectively 


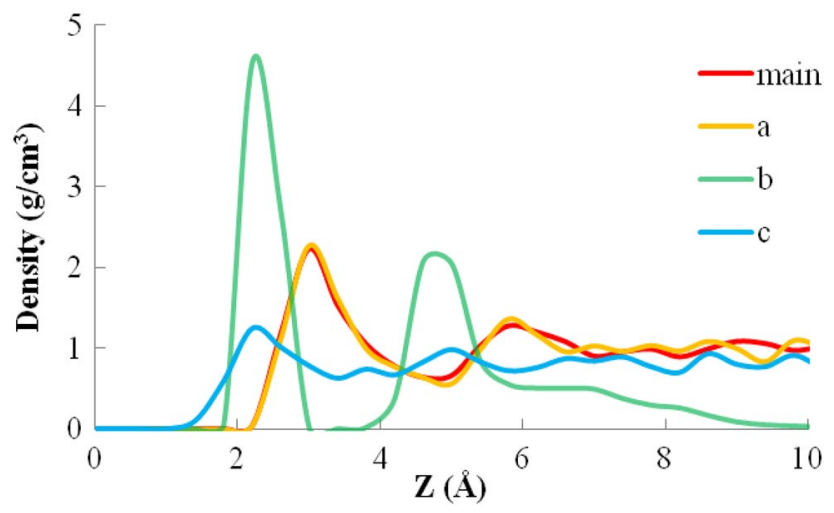

Fig. 3 Water density profile along the $Z$ axis (perpendicular to the substrate)

addition, from this comparison, it can be observed that the partial charge has not a considerable influence on the water interactions with the substrate. Recently, several publications have reported that the water molecules have weak electrostatic interactions with the molybdenum disulfide substrate $[17,19]$.

Comparison of systems $\mathrm{b}$ and $\mathrm{c}$ indicates that the density of water molecules near the substrate in system $b$ is much greater than in system c. It should be noted that the only difference between system $b$ and $c$ is that in system $c$, the partial charges of Mo and S atoms were considered to be zero. The observations of Fig. 3, are exactly consistent with those of in the contact angle. In addition, it was shown that the contact angles in the main system and system a are about $95^{\circ}$ and $62^{\circ}$, respectively, representing the identical interactions between the water and substrate for the two systems. However, the contact angles for system $\mathrm{b}$ and $\mathrm{c}$ are $18^{\circ}$ and $114^{\circ}$, respectively, but pointing out to the fact that the interactions between water and substrate is different for these two systems.

The relations between density, van der Waals and electrostatic interactions are also important. In the present studied system, the van der Waals interaction has direct relationship with the water molecules density. This means that the higher the density of the water molecules, the stronger the van der Waals interaction between the water and the substrate. On the other hand, there is no direct relationship between the electrostatic interactions of watersubstrate and water density. This is mainly due to the fact that the both positive and negative charges increase with an increment in the water density and they eliminate the effect of each other. Thus, in the electrostatic interactions, the density of positive and negative charges in different layers of the droplet is very important rather than of the water molecules. Figure 4 plots are the electrical charge changes of the drops in the $Z$ direction (perpendicular to the surface of the substrate) for the systems under study.

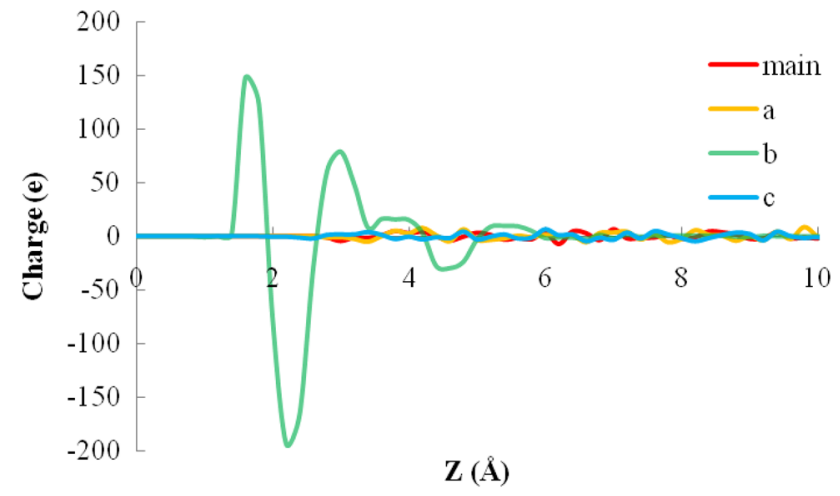

Fig. 4 The electrical charge variations of the drops in the $Z$ direction (perpendicular to the surface of the substrate) for the studied systems

Figure 4 illustrates that the distribution of the electrical charge along $Z$ direction for the main system, system a and $\mathrm{c}$ is close to zero while it highly fluctuates in the case of system $b$. To explain the above observation, the following reasons are presented:

The strong interactions between the water molecules near the surface causes the dipole vector of these molecules to be parallel to the surface, while the interaction between the water molecules and substrate causes this vector to be perpendicular to that. In the main system, system a and c, the dipole vector of water molecules deviates from being parallel to the surface as the interactions between water molecules is stronger than those between water molecules and substrate. Water molecules tend to be parallel to the surface. Therefore, the water molecules in the first layer close to the substrate are placed on a plane with almost identical $Z$. In this case, the total electrical charge between the water molecules close to the surface of the substrate tend to be in zero.

However, there is a strong interaction between water and substrate in system $b$ (the density diagram and water contact angle also confirm this theorem). Therefore, the water molecules in the layer near the substrate, deviate from the parallel mode and the total charge will not be in zero more along the $Z$ direction. Figure 5 shows the snapshot of the main system of water droplet and system $b$ as well as.

Figure 5 is in fact an indicative of the issues that discussed above. It can be seen from this figure that the water molecules in system $b$ are closer to the surface. This allows the hydrogen atoms to penetrate the surface more efficiently and interact with the surface sulfur atoms. As a result of this interaction, the positively charged water molecules (hydrogen) are placed closer to the surface than the negatively charged ones (oxygen). The explanations of Fig. 5 is in line with the observations of Fig. 4 corresponding to system $b$ and is in fact the molecular justification for Fig. 4.

It should be noted that the only difference between the two compared systems in Fig. 5 is that the sigma value (van 


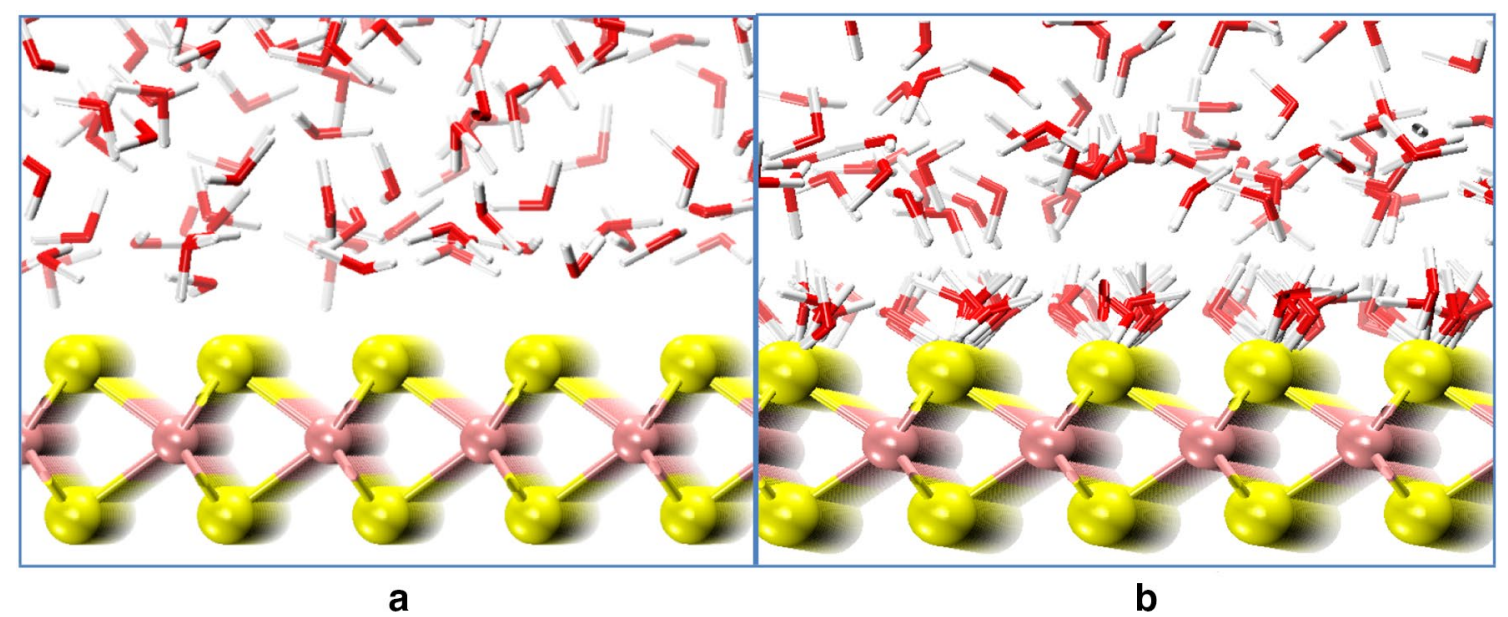

Fig. 5 Comparison of the droplet snapshot of the main system (a) and system b (b)

der Waals equation) in system $b$ is less than that of the main $\mathrm{MoS}_{2}$ system to the amount of $1 \AA$. It was shown in above sections that the electrostatic interaction plays a minor role in the water wettability of the main system. However, in system $\mathrm{b}$, this interaction plays a major role in the wettability. It can be concluded from these arguments that "the effectiveness of the electrostatic interactions on the wettability, depends on the sigma in the Lennard-Jones equation". In other words, "the roles of the electrostatic and van der Waals interactions on the wettability are not indissociable from each other".

\section{Conclusion}

In this study, it was shown that the charge variation and sigma of Lennard-Jones equation play significant roles in the wettability. The relationship between the variations of water density near the substrate and sigma changes is different from that with charge changes. The water density peak increases by reducing the sigma of Lennard-Jones. However, these changes are not observed for the time changes. The main conclusion of this work is that the wettability and contributions of the electrostatic and van der Waals interactions cannot be separated from each other.

Open Access This article is licensed under a Creative Commons Attribution 4.0 International License, which permits use, sharing, adaptation, distribution and reproduction in any medium or format, as long as you give appropriate credit to the original author(s) and the source, provide a link to the Creative Commons licence, and indicate if changes were made. The images or other third party material in this article are included in the article's Creative Commons licence, unless indicated otherwise in a credit line to the material. If material is not included in the article's Creative Commons licence and your intended use is not permitted by statutory regulation or exceeds the permitted use, you will need to obtain permission directly from the copyright holder. To view a copy of this licence, visit http://creativecommons.org/licenses/by/4.0/.

\section{References}

1. Stone, H.A., Stroock, A.D., Ajdari, A.: Engineering flows in small devices: microfluidics toward a lab-on-a-chip. Annu. Rev. Fluid Mech. 36, 381-411 (2004)

2. Squires, T.M., Quake, S.R.: Microfluidics: fluid physics at the nanoliter scale. Rev. Mod. Phys. 77(3), 977 (2005)

3. de Groot, B.L., Grubmüller, H.: Water permeation across biological membranes: mechanism and dynamics of aquaporin-1 and GlpF. Science 294(5550), 2353-2357 (2001)

4. Prabhu, K.N., Fernades, P., Kumar, G.: Effect of substrate surface roughness on wetting behaviour of vegetable oils. Mat. Des. 30(2), 297-305 (2009)

5. Wang, Y., Song, R., Liu, J.-J., Cui, M.-M., Ranjith, P.: Pore scale investigation on scaling-up micro-macro capillary number and wettability on trapping and mobilization of residual fluid. J. Contam. Hydrol. 225, 103499 (2019)

6. Dong, Y., Huang, C., Yang, X.-Y.: Underwater superoleophobic and underoil superhydrophobic surface made by liquid-exfoliated $\mathrm{MoS}_{2}$ for on-demand oil-water separation. Chem. Eng. J. 361, 322-328 (2019)

7. Sakai, M., Yanagisawa, T., Nakajima, A., Kameshima, Y., Okada, K.: Effect of surface structure on the sustainability of an air layer on superhydrophobic coatings in a water-ethanol mixture. Langmuir 25(1), 13-16 (2008)

8. Son, Y., Kim, C., Yang, D.H., Ahn, D.J.: Spreading of an inkjet droplet on a solid surface with a controlled contact angle at low Weber and Reynolds numbers. Langmuir 24(6), 2900-2907 (2008)

9. Perelaer, J., Hendriks, C.E., de Laat, A.W., Schubert, U.S.: One-step inkjet printing of conductive silver tracks on polymer substrates. Nanotechnology 20(16), 165303 (2009)

10. Gajrani, K.K., Suvin, P., Kailas, S.V., Mamilla, R.S.: Thermal, rheological, wettability and hard machining performance of $\mathrm{MoS}_{2}$ and $\mathrm{CaF}_{2}$ based minimum quantity hybrid nano-green cutting fluids. J. Mat. Proc. Technol. 266, 125-139 (2019) 
11. Ohler, B., Langel, W.: Molecular dynamics simulations on the interface between titanium dioxide and water droplets: a new model for the contact angle. J. Phys. Chem. C 113(23), 1018910197 (2009)

12. Song, Z., Zhang, Y., Liu, C., Xu, B., Qi, F., Yuan, D., Pu, S.: Insight into $\mathrm{OH}$ and $\mathrm{O}_{2}$-formation in heterogeneous catalytic ozonation by delocalized electrons and surface oxygen-containing functional groups in layered-structure nanocarbons. Chem. Eng. J. 357, 655-666 (2019)

13. Darvishi, M., Foroutan, M.: Molecular investigation of water adsorption on $\mathrm{MoS}_{2}$ and graphene surfaces. J. Mol. Liq. 225, 1-10 (2017)

14. Heyne, M., Chiappe, D., Meersschaut, J., Nuytten, T., Conard, T., Bender, H., Huyghebaert, C., Radu, I.P., Caymax, M., de Marneffe, J.-F.: Multilayer $\mathrm{MoS}_{2}$ growth by metal and metal oxide sulfurization. J. Mat. Chem. C 4(6), 1295-1304 (2016)

15. Hiratsuka, M., Emoto, M., Konno, A., Ito, S.: Molecular dynamics simulation of the influence of nanoscale structure on water wetting and condensation. Micromachines 10(9), 587 (2019)

16. Darvishi, M., Foroutan, M.: Molecular investigation of oilwater separation using PVDF polymer by molecular dynamic simulation. RSC Adv. 6(78), 74124-74134 (2016)

17. Foroutan, M., Fatemi, S.M., Darvishi, M.: Formation and stability of water clusters at the molybdenum disulfide interface: a molecular dynamics simulation investigation. J. Phy. Condens. Matter 30(41), 415001 (2018)
18. Foroutan, M., Darvishi, M., Fatemi, S.M., Babazadeh, K.H.: Water chain formation on rutile $\mathrm{TiO}_{2}$ (110) nanocrystal: a molecular dynamics simulation approach. J. Mol. Liq. 250, 344-352(2018)

19. Liu, C., Jin, Y., Li, Z.: Water transport through graphene and $\mathrm{MoS}_{2}$ nanopores. J. Appl. Phys. 126(2), 024901 (2019)

20. Plimpton, S.: Fast parallel algorithms for short-range molecular dynamics. J. Comp. Phys. 117(1), 1-19 (1995)

21. Humphrey, W., Dalke, A., Schulten, K.: VMD: visual molecular dynamics. J. Mol. Gr. 14(1), 33-38 (1996)

22. Hoover, W.G.: Canonical dynamics: equilibrium phase-space distributions. Phys. Rev. A 31(3), 1695 (1985)

23. Lennard-Jones, J.E.: Cohesion. Proc. Phys. Soc. 43(5), 461 (1931)

24. Abascal, J.L., Vega, C.: A general purpose model for the condensed phases of water: TIP4P/2005. J. Chem. Phys. 123(23), 234505 (2005)

25. Sheng, S., Fu, J., Wong, B.M., Wu, J.: Solvation structure of surface-supported amine fragments: a molecular dynamics study. J. Phys. Chem. C 121(40), 22156-22163 (2017)

Publisher's Note Springer Nature remains neutral with regard to jurisdictional claims in published maps and institutional affiliations. 